\section{EMBRYRIDDLE \\ Aeronautical University}

SCHOLARLY COMMONS

\section{International Journal of Aviation,} Aeronautics, and Aerospace

$10-1-2014$

\title{
Interactive 3-D Software in Aircraft Rescue and Fire Fighting Training
}

Mary K. Gorman Ph.D.

Embry-Riddle Aeronautical University, Worldwide, gormanm@erau.edu

Rita "Rene" I. Herron Ph.D.

Embry-Riddle Aeronautical University, Worldwide, herronr1@erau.edu

Follow this and additional works at: https://commons.erau.edu/ijaaa

Part of the Emergency and Disaster Management Commons

\section{Scholarly Commons Citation}

Gorman, M. K., \& Herron, R. I. (2014). Interactive 3-D Software in Aircraft Rescue and Fire Fighting Training. International Journal of Aviation, Aeronautics, and Aerospace, 3(2). https://doi.org/10.15394/ ijaaa.2016.1031

This Article is brought to you for free and open access by the Journals at Scholarly Commons. It has been accepted for inclusion in International Journal of Aviation, Aeronautics, and Aerospace by an authorized administrator of Scholarly Commons. For more information, please contact commons@erau.edu. 
The aircraft rescue and firefighting (ARFF) profession is vital to emergency response at airports in the United States and abroad. Mandated by the Federal Aviation Administration (FAA), regular training of these professionals includes a targeted set of both hands-on and classroom components. The Dallas/Fort Worth (DFW) Fire Training Research Center (FTRC) has developed the first technologically-advanced software to assist in the ARFF pedagogy. It is a new learning approach to provide ARFF professionals with a three-dimensional (3-D) virtual reality platform comprised of airport familiarization, aircraft familiarization, and strategies and tactics learning modules. Through a 2013 Memorandum of Understanding, the world's leading aviation and aerospace university, EmbryRiddle Aeronautical University (ERAU), and DFW FTRC have created a unique partnership for the conduct of research to advance ARFF education and explore emerging issues in the discipline.

\section{Significance of the Study}

At the DFW FTRC, ARFF students can use the software to explore 3-D airport runways for airport familiarization, and digital models of individual aircraft and their internal operating systems. The instructors can develop complex response scenarios using the strategies and tactics module. Instructors have the option to allow students to work individually, in collaboration with colleagues, and with the DFW FTRC instructors in the classroom. The employment of this technological tool has significant implications for the training of ARFF personnel worldwide. It also has the potential to translate across public safety programs containing inherent risk as a component of training.

\section{Purpose Statement}

As the nexus between aviation, fire science, and emergency management becomes increasingly more complex and costly, technology tools can be used to augment training, defray cost and avoid risk while maintaining authenticity with situational reality. The manner in which the learning content is effectively imparted to the student-professional can be the difference between saving lives and a disastrous emergency response.

In 2013, the DFW FTRC used the Survey Monkey instrument to assess verbal feedback it had been receiving from students. Results from this unscientific study confirmed positive student perceptions of the software's utility for augmenting hands-on training, and also suggested more uses for the software, and a wider distribution if it could be made affordable. Although the data was not scientific and not quantitatively analyzable, it led to more questions. In the tradition 
of qualitative research, this exploratory study was designed to elucidate, and add depth and meaning to student perceptions of the educational experience with the software (Patton, 2002, p. 193).

\section{Literature Review}

The research examined the use of 3-D virtual reality software at the DFW FTRC training facility. This type of technology is not being used at any other ARFF training facility, therefore, similar research measuring the efficacy of this training method in this context does not exist in the literature. A review of ARFF training literature was performed, as this would be the training framework within which the software and training curriculum is operationalized. United States (US) 14 CFR Part 139, sections 315 through 321, mandate the operational and training requirements for ARFF as the baseline for annual and recurrent training, as overseen by the Federal Aviation Administration (FAA). These include annual requirements for on-site airport firefighters, including "live burn" training and emergency medical capabilities (FAA, 2011). Various FAA Advisory Circulars issued periodically under Part 139 provide more detailed guidance, as necessary, to address training, equipment, and operational aspects of the ARFF profession. The National Fire Protection Administration (NFPA) publishes national consensus documents which are widely used to guide professional development in the industry. NFPA 1003 (2015 edition), the Standard for Airport Firefighter Professional Qualifications and NFPA 405 (2015 edition), the Standard for Recurring Proficiency of Airport Firefighters are the two main documents providing initial and recurrent training guidelines as well as tactical and strategic guidance for professionals. The International Fire Service Training Association (IFSTA) $\left(5^{\text {th }}\right.$ edition) ARFF Manual is the cornerstone textbook in the ARFF profession, used for the majority of certification testing in the industry. What these publications revealed is that baseline training standards have been mandated by the FAA; other than these, measures of operational proficiency and professional development are merely suggested in the literature.

The effectiveness of flight simulators for pilot training has been studied extensively by NASA and the U.S. Air Force since the 1940s and 1950s. Adams' "Some Considerations in the Design and Use of Dynamic Flight Simulators," published in the "Fidelity of Simulation and Transfer of Training," was one of the first studies of haptic skill transfer in training (Gerathewohl, 1969). Since then, pilot training using simulators has evolved with time and technology. A study by Macchiarella, Arban, and Doherty (2006) at ERAU's Daytona Beach campus confirmed a positive skill transfer using simulated flight as a primary means of training pilots from novice to certification. Similarly, a study by Reweti (2014) 
evaluated personal computer-based pilot training for visual flight rules procedures in a comparative study of training effectiveness. In this research, results suggested that the interactive component of student-instructor emerged as significant, integration into curriculum was central to student success, and positive psychomotor skill transfer was observed for repetitive tasks.

Although pilot training using simulators is similar, the ARFF training at DFW FTRC is arguably more fully-physical and less repetitive in nature. Therefore, the literature review was expanded to include "interactive software" and "hands-on training" studies in other professional applications. The literature suggested that, generally, positive results have been measured in business, manufacturing, communications, medical, and security industries, but little direct transferability from this research was deemed appropriate. However, a 2003 study measuring the learning outcomes of electronics engineering students using a computer simulator versus a hands-on trainer found that frequency of access, "motor characteristics," and memory reinforcement emerged as key factors among posttest measures (Kulatunga, 2003, p. 37). This study was considered transferable because the hands-on assessment of student proficiency was comprised of: non-repetitive physical action, independent problem-solving, and assessment immediately followed several weeks of classroom instruction. The research design and assessment of student knowledge and haptic skill transfer were similar to this study. In contrast, but also considered transferable, a 2013 study of rowing determined that virtual reality simulator training had a statistical advantage over training onwater; this study used biomechanical measures, both through a questionnaire of participants and as evaluated in video by a rowing expert (blinded to the group) (Rauter et al., 2013). Findings from this study are significant because ARFF is largely a fully-physical profession and the transfer of haptic skills (from the classroom to the field training environment) is precisely how the instructors at DFW FTRC assess student proficiency connected to software effectiveness. The Kulatunga and Rauter et al. studies suggested that virtual reality learning environments, similar to the DFW FTRC, created a positive basis for both the conveyance of knowledge and physical skills from the classroom to the field.

\section{Methodology}

DFW FTRC is the only training facility employing this software and this technology, however, the effectiveness of this experiential learning had not been formally studied. The MOU between ERAU and the DFW FTRC enabled a collaborative research effort to be undertaken to explore the following:

Research Question: How effective is the virtual technology software in 
translating knowledge and accelerating haptic skill transfer to the hands-on portion of ARFF training at the DFW FTRC?

In accordance with Yin (2012), the single-case case study method was selected for this exploratory research as the DFW FTRC is both a "critical case" (crucial to the understanding of the research question), and a "revelatory case" (previously inaccessible opportunity) (p. 51-52).

Data collection for this case was comprised of both classroom observations (two classroom observations) and semi-structured interviews of purposefullyselected key informants within their natural setting. Questions for the key informant interviews were developed after the classroom observation portion of the study and from the conceptual framework. As with most qualitative research, data collection and analysis occurred concurrently. Clear and consistent research questions and interview protocols were employed (provided in the Appendix), and peer review was used throughout the research process. This approach was taken to ensure subject matter saturation, establish the importance of context, use of multiple forms of data and multiple researchers for triangulation, provide interpretation of participant meaning and a full accounting of the complex issue being studied-all consistent with qualitative methods (Creswell, 2015).

In accordance with Yin's (2012) criteria for the single-case study, validity was achieved through the use of multiple sources of evidence (triangulation), multiple perspectives (investigator triangulation), establishing a chain of evidence, and key informant confirmation of data collected. Ensuring coherence through phased coding, inter-rater reliability, subject matter saturation, pattern matching, multiple methods (classroom observation and key informant interviewing), explanation building and addressing rival explanations were also performed to confirm credibility, or internal validity (Miles \& Huberman, 1994; Yin, 2012). According to Lincoln \& Guba, (1985), transferability of the research findings must be evaluated by the reader; however convergence and divergence of the data was used to contextualize findings and assess external validity (Yin, 2012). Reliability and confirmability of the research were achieved by adherence to case study protocols, development of a case study database in NVivo 10, triangulation of methods and data sources (Yin, 2012).

\section{Conceptual Framework}

The initial conceptual framework for this study was constructed of a priori themes from the 2013 DFW FTRC quantitative survey, theories contributed by key informants before the interview process, and from the classroom observation 
portion of the study. Concepts from the DFW FTRC survey and key informants included: "fear of technology" and "age/generational." From the first phase of the research (classroom observations) "integration into the curriculum" and "interactive" were added to the conceptual framework. These four a priori themes thus comprised the initial conceptual framework and preliminary nodes for data analysis.

\section{Data Collection}

\section{Classroom Observations}

Researchers were non-participant observers during two classroom sessions at the DFW FTRC facility so that both the student and instructor actions could be recorded in real-time. The observations were done at two class sessions of the Basic Aircraft Rescue and Fire Fighting (BA-120) course. The course is 120 hours total, given over a three week period; material is reviewed in the classroom and handson training in the field is conducted in the specified topic areas immediately following classroom instruction.

First Classroom Observation. The class was taught by two male DFW FTRC instructors. The class consisted of 12 students (10 male, 2 women), ages ranging from late-20s to early-50s, from different small and regional-sized airports from various states.

The lecture portion of the course was completed after 3 hours, comprised of PowerPoint (PPT) slides and textbook-focused, with the afternoon dedicated to the hands-on training. The instructors used PPT slides and continuously referred students to the textbook while lecturing. They mentioned the software a total of six times, but did not actually utilize it. The software portion of the class only lasted 45 minutes, but students were encouraged to practice it during free times: breaks, lunch, etc.

We observed that older students had difficulty with software maneuverability issues, suggesting unfamiliarity with iPad techniques. The students who were younger often leaned over to assist, or one of the instructors would come to aid the students. The majority of the class entered software module areas easily and immediately, but hesitated using the touch screen capabilities (e.g. rotating, zooming). There were two students who had no idea of how to use a touch screen. 
On the second day, the software portion was used after the initial lecture which lasted for 1.5 hours. The software was used for 40 minutes and was part of the students' aircraft familiarization.

We observed a majority of the students exhibited more confidence and an increased level of familiarity with the software on the second day. During the observational time, there were two students who took advantage of using the software when class was not in session. These students independently went into the strategies and tactics module and created situations on the virtual runway.

Second Classroom Observation. The second class was taught by a different male instructor (not observed previously) teaching alone. The class was consisted of 11 students (all male), ages ranging from mid-20s to mid-50s, all Texas firefighters from Dallas, Fort Worth, Denton and McKinney departments. The observation of the class began during the aircraft familiarization part of the course. The first use of the software came 1.5 hours into the class after lectures utilizing PPTs and the textbook.

We observed that all but two students immediately opened the software and became engaged in exploration. There was some difficulty in navigation, but students did not hesitate to ask for assistance at various times to verify that they were in the correct location within the software module. There were two older students who did not touch their computers at all throughout the interactive software portion of the course; instead, they moved their chairs closer to fellow students and watched them go through the exercise with the instructor. In comparison to the previous class, this instructor took more time to demonstrate maneuverability of the software.

On the second day, students used the strategies and tactics portion of the software after 1.5 hours of lecture. The instructor used the virtual runway and placed obstacles (construction barriers, apparatus, tractors and additional aircraft) on the screen and then allowed the students to generate their own scenarios. The instructor stopped students after 15 minutes of work and drew a circle around some icons on the smart board. Students attempted to do this on their monitors, but could not because that option was not available on student monitors (circling is a smart board feature and not a software option). Once the instructor realized this, he created a table top exercise for the class.

Students who did not previously engage with the software were assisted by their younger colleagues on navigational aspects and adding aircraft and obstacles to scenarios. Again, it appeared that student confidence increased with familiarity on the second day. Researchers noted completely different instructor integration 
and time using the software, despite this being the same course observed in the first classroom observation.

Classroom Observation Conclusion. DFW FTRC's a priori theme of "age/generational" differences was confirmed, however, the "fear of technology" concept was more accurately characterized as student familiarity and confidence with the software. From the classroom observations, the themes of "integration into the curriculum" and "interactive" also emerged. It was evident between the two classroom observations that separate instructors at the DFW FTRC use (and thus integrate) the software differently, even within the same course; this suggested a topic for further exploration and discussion with the key informants.

\section{Key Informant Interviews}

Key informants were purposefully selected for their knowledge about training at the DFW FTRC. At the time the interviews were conducted, there were a total of five instructors at the facility using the software in courses; key informant interviews comprised four of these instructors, summarized in Table 1.

Each instructor had direct contact with students in both the classroom and the hands-on field portion of the training. Thus, they were able to observe how the knowledge imparted in the classroom did/did not readily transfer to the field by their firsthand observations of student performance.

All interviews were privileged and conducted on-site at the DFW FTRC in

a private meeting room. Confidentiality of responses was ensured in writing. Data collection was comprised of semi-structured interviews in accordance with a predetermined interview protocol (Appendix) and audiotaped using an Olympus VN702PC digital voice recorder; note taking was performed by each researcher.

\section{Data Analysis}

Interview transcripts were transcribed into text format and entered into NVivo 10 qualitative data analysis software as internal Microsoft Word documents. In accordance with Yin's (2012) guidance for case study, classroom observation notes taken by both researchers and interview transcripts were read several times to ensure immersion in the data. An iterative process returning to the conceptual framework provided a focused analysis, increased congruence, precision of pattern matching, and strengthened internal validity (Yin, 2012). 
Table 1

Key Informant Summary Information

\begin{tabular}{cccl}
\hline Key Informant & $\begin{array}{l}\text { Professional Fire } \\
\text { Experience (Years) }\end{array}$ & $\begin{array}{l}\text { DFW FTRC } \\
\text { Experience } \\
\text { (Years) }\end{array}$ & Rank/Job Title \\
\hline Key 1 & 6.5 & 2 & $\begin{array}{l}\text { Lead Instructor } \\
\text { Supervisor }\end{array}$ \\
"No Bias" & 20 & 2 & Training Chief \\
Key 2 \\
"No Bias \\
Key 3
\end{tabular}

The a priori themes from the conceptual framework represented the initial nodes created in the NVivo 10 software, whereas codes are pieces of data (text) from the interview transcripts and classroom observation notes which relate directly to these larger concepts. Additional nodes were added as super-ordinate (primary nodes) and sub-ordinate themes (child nodes) developed during the data analysis process.

Key Informant Interview Results. A review of the interview responses will demonstrate how the codes support the emergence of themes during the data analysis process, and respondent quotations will support the importance of these themes. Interview questions are provided in the Appendix. Table 2 summarizes the super-ordinate and sub-ordinate themes, with the number of corresponding codes occurring within the data provided by key informant source. This review will also demonstrate how the nodes connected from the interview transcripts to the conceptual framework, connected super-ordinate themes, and how patterns emerged supporting the development of sub-ordinate themes.

Key informants were asked what they observed in the level of difficulty students experienced using the software. Instructors immediately described the "characteristics" of students, demographically, culturally, according to age, and as 
ARFF professionals (hierarchy, rank, and as separate from the rest of the fire service). Three of the four key informants used the word "generation" in answering this question, and they described how younger students are more comfortable with the technology than older students. This was consistent with the a priori theme of age/generational in the conceptual framework. One informant described, "Young groups pick up much quicker than the older generation. Problems I've seen are people being unfamiliar with using the computer touch screen, learning how to function in a Windows environment, following along with the instructor-age deviation."

Table 2

Emergent Themes by Interview

\begin{tabular}{lccccc}
\hline Themes & Key 1 & Key 2 & Key 3 & Key 4 & Total \\
\hline Culture & 8 & 5 & 9 & 9 & 47 \\
$\quad$ ARFF & 0 & 3 & 7 & 7 & 17 \\
$\quad$ Learning & 8 & 2 & 2 & 2 & 14 \\
$\quad$ Age/Generational & 3 & 2 & 3 & 1 & 9 \\
$\quad$ International & 1 & 4 & 2 & 0 & 7 \\
Time & 7 & 2 & 4 & 14 & 27 \\
Interactive & 7 & 8 & 5 & 6 & 26 \\
Integration/Curriculum & 7 & 2 & 4 & 9 & 22 \\
Memory & 5 & 3 & 1 & 4 & 13 \\
Fear of Technology & 1 & 2 & 3 & 3 & 9 \\
\hline
\end{tabular}

As coding in this category continued, the age/generational theme figured to be sub-ordinate, and "culture" was determined to more accurately characterize the super-ordinate theme. Significant differences were also noted between domestic and international (sub-ordinate) students pertaining to the level of excitement about using the software and aptitude. While international students may not have had experience with this specific technology previously, the instructors believe they were able comprehend the software and touch screens more readily than domestic students. One of the key informants remarked: "The high tech software is a major 
draw from our international students, Beijing, Shanghai. We just got through with a class from South Africa-they thought it was the bomb."

Also emerging as sub-ordinate, the theme of ARFF was identified as significant with regard to acceptance of this technology. Traditional emergency services' training is comprised of lecture, PPT and tabletop exercises. One key informant described the software as "a distraction" to teaching, and felt it should only be used to "reinforce" lecture because that is what students are "used to." The a priori fear of technology theme was overwhelmingly connected with this part of the interview. Comments were connected to instructors, however, and not students; key informants discussed the hesitancy on the part of their colleagues to embrace technology in training and to integrate software into existing curriculum.

Learning emerged as another sub-ordinate theme connected to difficulty students had with the software, but this was associated with differences in individual learning and teaching styles. This concept included instructor strengths/weaknesses and how comfortable an instructor may be with the software. Also connected to the learning theme, topics included the instructional theories of adult and group learning as well as student pre-conceived ideas about the facility (DFW FTRC), the course, the material and using software.

The key informants were asked how well they believed the software was integrated into the curriculum and if they had recommendations for better leveraging opportunities for incorporating it into the learning environment. This a priori theme became part of the conceptual framework following the classroom observation portion of the research. In answering this question, all of the key informants addressed recommendations for integration of the software into the classroom and suggested future delivery methods. Interview responses revealed that the usage of the software was actually at the discretion of individual instructors, with no clearly defined integration guidance from curriculum designers.

It was at this point that the theme of time emerged as significantly connected to the learning culture and integration into the curriculum. Because the training classes must cover certain state and federal training requirements, the problem of having the time for software use in the classroom was mentioned by all key informants. Another aspect of time was that, although the software was created with the curriculum in mind, there has not been adequate time for the instructors to review the course material and designate how the software could be effectively integrated into the existing curriculum. Furthermore, instructors noted that they are not trained in curriculum and/or instructional design, putting them at a disadvantage in overlaying the software to already designed courses; while the software may 
have the potential to compliment classroom materials, it cannot if it is ineffectively implemented.

Observations of whether or not the software had an impact on the hands-on portion of training melded into responses about a belief if the software created a better learning experience for students. The a priori interactive theme was confirmed by key informants as they described how the software provides "progressive training," "enables them [students] to catch-on faster," "gives them the opportunity to think outside the box," and "puts things from every angle." Recognizing that student expectations change with advances in technology, key informants unanimously agreed that this software enables learners the flexibility to "touch and move things around" and experience the type of "immersive" training that "takes tabletop exercises off the table and puts them on the computer."

Further, key informants unanimously and emphatically agreed that the software positively impacted the hands-on portion of the training sessions. All four key informants mentioned that being able to learn from 3-D software rather than a PPT presentation created superior retention (memory theme). Instructors also stated that being able to simulate touch and vision provided a quicker learning curve, and this was obvious in the display of physical skills. One key informant stated:

Everything we do is graduated. You start out with the software and go out and play in a fire with an airplane and smoke, then chambers within fire, then aircraft mock up and everything and every step builds. Can't build a good house without having a good foundation and [the software] is a great starting place for students.

The significance of this portion of the interview demonstrated how the software helped solidify student knowledge by providing a connection between lecture and hands-on training in advance of training. This is consistent with Kulatunga's 2003 study of engineering students, which discovered a statistically significant difference between students working in virtual reality versus hands-on trainers in the area of "reinforcing thought processes" (p. 37) prior to performing motor characteristics testing. This research suggested that the frequency of student access was an advantage through reinforcing memory and thought processes. Further, the research suggested that simulators can be used effectively to transfer knowledge, reduce costs, and save classroom time in electrical engineering programs with a hands-on learning component (Kulatunga, 2003, p. 38).

Similarly, the 2013 Rauter, et al. study of rowing skill transfer using a 3-D virtual reality-based simulator found statistically significant skill gains in both 
initial skill levels of participants and advanced skill improvements. The study used 10 technical aspects of biomedical performance rated by participants in a questionnaire (21 questions) and independently evaluated using video evidence rated by a rowing trainer (blind reviewer). "Realism was confirmed," as was performance consistency, and study participants "agreed that the simulator offers a clear advantage" by being able to focus on single, technical aspects of the task (Rauter, et al., 2013, p. 16). Opposing ratings (between participant and blind reviewer) were only found in two of the cases. The significance of this study is that it supports the effectiveness of augmented virtual reality learning environments, similar to the DFW FTRC software, for "visual, auditory, and haptic interactions" skill gains (Rauter, et al., 2013, p. 16). The DFW FTRC software is used to impart knowledge as well as accelerate specific visual, auditory, and haptic skill transfer from the ARFF classroom to the hands-on portion of training.

Key informants were also asked to describe the survey of student attitudes that the DFW FTRC conducted in 2013, which returned a 55\% preference for the strategies and tactics module, followed by aircraft familiarization at $37 \%$. The instructors were specifically asked their opinion of the survey responses for preferences on "how a department/agency would utilize the software." The interactive theme came forward, as the software can create site-specific scenarios, input individual airport landing strips from Google Earth, move or delete obstructions, aircraft and apparatus. The aircraft familiarization is believed to be favored second by students because they can view "simulated aircraft" that they may never see, as the majority of airports that send personnel to FTRC are smaller in size (DFW FTRC, 2013).

Key informants were also asked if this type of software should be part of ARFF training no matter where the students received their training. Three out of four key informants did not hesitate to respond that they believe it would be extremely beneficial to all ARFF personnel, while one questioned where it might "fit" it into the required curriculum and emphasized the need for traditional lecture instruction.

Finally, the key informants commented that students from different airports and various federal, state and local agencies have all expressed interest in the software, specifically the strategies and tactics module. This has resulted in the DFW FTRC having to respond to many requests for the software to be marketed in tabletop and tablet forms. Looking forward, one key informant summarized the software as "the next stage of learning for an ARFF environment." 


\section{Classroom Observation Data Analysis}

Classroom observation data were coded after the interview data because additional nodes (to supplement the conceptual framework) could be gleaned from key informant interviews. The occurrence of codes by node according to source is provided in Table 3 for the classroom observation notes and summaries made by each researcher (Gorman and Herron) for each of the two classroom observations (November, 2013, January, 2015). Each observer is referred to as "A" and "B."

Table 3

Emergent Themes from Classroom Observation Notes

\begin{tabular}{lccccc}
\hline Themes & A Obsv1 & A Obsv2 & B Obsv1 & B Obsv2 & Total \\
\hline Culture & 3 & 0 & 7 & 0 & 17 \\
$\quad 1$ & 0 & 1 & 0 & 2 \\
$\quad$ ARFF & 2 & 0 & 6 & 0 & 8 \\
$\quad$ Learning & 3 & 1 & 3 & 0 & 7 \\
$\quad$ Age/Generational & 0 & 0 & 0 & 0 & 0 \\
$\quad$ International & 1 & 3 & 10 & 2 & 16 \\
Integration/Curriculum & 1 & 1 & 8 & 1 & 11 \\
Time & 3 & 2 & 1 & 2 & 8 \\
Interactive & 1 & 2 & 4 & 0 & 7 \\
Fear of Technology & 1 & 2 & 1 & 0 & 4 \\
Memory & & & & & \\
\hline
\end{tabular}

From the classroom data, themes of culture, integration into the curriculum, and time were coded most often in the data. The theme of culture focused on learning culture, primarily suggested improvements to the overall learning environment and instructional culture. These encompassed software, curriculum, and integration aspects of experiential learning, as well as consistency of delivery. Age/generational differences among the students was clearly observed (verified a priori concept) in the quality/quantity of student interactions with the software, participation in class, and overall perception of student engagement. Researchers noted that ARFF culture appeared to influence training, principally with regard to these professionals having an overall acceptance of new technology training tools 
as compared to hands-on and traditional methods of training (e.g. lecture, PPT, tabletop). Researchers also observed that the software was not well-integrated into the curriculum, there were differences among instructors and between classes (learning sub-ordinate theme), and the software's capabilities could be better leveraged during class time. As regards the theme of time, researchers commented that if students were able to use the software more, outside of the classroom, familiarity and navigation skills could be improved, competency increased, which would lead to more effective use of instructor time.

The interactive theme was observed with how actively the students engaged with the software, and researchers also noted the overall interactive behaviors among students and between instructor and class (as it pertained to the software, specifically). The a priori theme, fear of the technology, was considered as behavior associated with apprehension engaging with the software, alternatives to engagement, which students exhibited the behavior and specific activities that were problematic. This was observed with the older students and navigational aspects of the software (rotation, menu). The memory theme was less prevalent in observations (as compared to key informant interviews), but was recorded as related to student recognition of concepts as demonstrated by proficiency.

\section{Conclusion}

The research question, how effective is the virtual technology software in translating knowledge and accelerating haptic skill transfer to the hands-on portion of ARFF training at the DFW FTRC, was approached using a qualitative, exploratory research strategy. This single-case study was comprised of both classroom observations and interviews with subject matter experts having firsthand knowledge of student performance for their assessment of haptic skill transfer.

Table 4 provides a summary of the conceptual framework themes derived from this research. The culture theme was super-ordinate when combining data from both observation and key informant interviews; this suggests that, when assessing performance, common individual student characteristics emerged as significant. Sub-ordinate themes comprised of learning, ARFF, age/generational, and international suggest that there are notable variations in individual characteristics comprising professional, demographic, and environmental dimensions that may influence student proficiency and attitudes. The research found that international and younger students were both more enthusiastic and more proficient in using the software. ARFF students have some bias and hesitation about the software as they transition from traditional tabletop and lecture methods to a virtual reality learning environment. No variations were observed or noted in 
gender or professional rank among the students. The exception to individual student characteristics under the super-ordinate theme is the learning theme, related to the learning environment and instructional culture. These data revealed that significant variability exists among instructors and individual classes, which represents an internal training and operational challenge. It also brought to the forefront the need for instructors to be both comfortable and proficient with technology, curriculum development, adult and group teaching concepts.

\section{Table 4}

Conceptual Framework Themes

\begin{tabular}{lccc}
\hline Themes & $\begin{array}{c}\text { Classroom } \\
\text { Observations }\end{array}$ & Interviews & Total \\
\hline Culture & 17 & 47 & 64 \\
ARFF & 2 & 17 & 19 \\
Learning & 8 & 14 & 22 \\
Age/Generational & 7 & 9 & 16 \\
International & 0 & 7 & 7 \\
Time & 11 & 27 & 38 \\
Integration/Curriculum & 16 & 22 & 38 \\
Interactive & 8 & 26 & 34 \\
Memory & 4 & 13 & 17 \\
Fear of Technology & 7 & 9 & 16 \\
\hline
\end{tabular}

Time and integration/curriculum combined equally to be the second most notable themes of the research. This result is not surprising, as the concerns expressed by key informants were mainly not having enough time to devote to properly integrating the software into the curriculum, and the researchers observed the same in the classroom. Time was also a concern with curriculum design, achieving state/federal learning objectives, and the time necessary for instructors to adequately prepare for classes having the software as a central component (hardware preparation and troubleshooting). Key informants believed that having administrative support, additional personnel dedicated to software and curriculum 
development, and time built-in to instructor schedules for class preparation were all critical to the success of increasingly integrating the software into the curriculum.

The interactive qualities of the software were the most affirming aspects in reference to design and intent (as interactive 3-D software), observed both in the classroom and as described by key informants. The increased ability of students to interact with each other, with the instructors, and with the material is the cornerstone of this software and the DFW FTRC curriculum. This suggests that interactive software for ARFF has positive potential if it can be effectively integrated into mandated curriculum.

The theme of memory was mentioned more often by key informants as compared to researchers in classroom observations because it was positivelyassociated with the transference of knowledge and physical skills from the classroom to the hands-on portion of training. Classroom observers did not have the opportunity to see the hands-on portion of training. Finally, the fear of technology was among the concerns of the key informants and DFW FTRC administrators (from the 2013 survey) and was observed in the classroom, but was the least coded theme in the data; this suggests it was not as prevalent as originally believed. The research suggests that fear of technology may be more accurately described as a student's ability to become familiar and comfortable with the software, as seen on the second day of classroom observations.

Moving forward with the next generation of the software, integration into the curriculum and interactive challenges could be improved by providing previous access to the software in advance of attending class - either online, in a preparatory course, or on iPads or tablets (which students can take with them while they attend class). This could help students increase familiarity with the software, thus helping master simple maneuverability and menu challenges, and improving student confidence and interest.

Software improvements noted by both key informants and researchers included: additional aircraft (smaller planes more common to a majority of students), pre-loaded airstrips, weather condition options, and extra smart board features. Having the ability to load a Google map for each individual class seamlessly, during class, would create a richer learning experience. In addition, varying weather conditions or incident conditions at any time during the class would create a variety of stimulating and challenging scenarios for students. 


\section{Recommendations for Future Research}

Recommendations for future research include assessing the benefits of experiential learning when applied to firefighter education and training. DFW FTRC's software and curriculum is one example of such an application. Future research may also evaluate pedagogy to categorize learning constructivism using simulator-based and software-supported education and training. How this may be applied to public safety professions where risk is an inherent part of training is a crucial need in the industry.

One key informant described the software as "a harbinger of future learning," and it is, in many ways. With the interest exhibited by other emergency services professionals, it has capability across security, emergency medical, and federal, state and local public safety agencies. The extent to which this may be transferable should be the topic of future research. As exhibited from the existing ARFF literature within which the software is operationalized, it has tremendous training potential if it can be made financially affordable to all departments - which remains an ongoing task for the DFW FTRC. If the software could be widely distributed, it opens up other training alternatives for the DFW FTRC, including online training options. This research encourages DFW FTRC's use of the software and curriculum in a positive capacity to translate knowledge and accelerate haptic skill transfer from the classroom to hands-on training. As we continue to improve innovative technology tools in this fast-paced ARFF profession, and across all of public safety, we look forward to doing so collaboratively, with our DFW FTRC partners. 


\section{References}

Adams, J.A. (1957). Some considerations in the design and use of dynamic flight simulators. AFPTRC-TN-57-51. Selected Papers on Human Factors in the Design and Use of Control Systems. Lackland Air Force Base, TX: Air Force Personnel and Training Research Center.

Baxter, P., \& Jack, S. (2008). Qualitative case study methodology: study design and implementation for novice researchers. The Qualitative Report, 13(4), 544-559. Retrieved from http://www.nova.edu/ssss/QR/QR13-4/baxter.pdf

Creswell, J.W. (2015). 30 Essentials for the Qualitative Researcher. (1 ${ }^{\text {st }}$ Ed.). Thousand Oaks, CA: Sage Publishers.

Dallas/Fort Worth Fire Training Research Center (2013). [Survey Monkey student and visitor perceptions]. Unpublished raw data.

Federal Aviation Administration. (2011). Code of federal regulations title 14 part 139: Aircraft rescue and firefighting: operational requirements. Retrieved from http://www.gpo.gov/fdsys/pkg/CFR-2011-title14-vol3/xml/CFR2011-title14-vol3-sec139-319.xml

Federal Aviation Administration. (2014). Addendum: Advisory circular number 150/5210-17C: programs for training of aircraft rescue and firefighting personnel. Retrieved from http://www.faa.gov/documentLibrary/media/Advisory_Circular/draft_150 _5210_17c.pdf

Gerathewohl, S. (1969). Fidelity of simulation and transfer of training: A review of the problem. Paper presented at AGARD-NATO--Advanced Operational Aviation Medicine. Retrieved from https://www.faa.gov/data_research/research/med_humanfacs/oamtechrepo rts/1960s/media/AM69-24.pdf

International Fire Service Training Association. (2008). Aircraft Rescue and Fire Fighting, 5th Edition. Stillwater, OK: International Fire Service Training Association.

Kulatunga, A. (2003). Learning outcomes of simulating hands-on activities for electrical motor controls through interactive software. Journal of Engineering Technology, 20(1), 34. 
Lincoln, Y. S., \& Guba, E. G. (1985). Naturalistic inquiry. Beverly Hills, CA: Sage Publications, Inc.

Macchiarella, N., Arban, P., Doherty, S. (2006). Transfer of training from flight training devices to flight for ab-initio pilots. International Journal of Applied Aviation Studies, 6(2), 299-314.

National Fire Protection Assocation. (2014). NFPA 403: Standard for Aircraft Rescue and Firefighting Services at Airports. Qunicy, MA: National Fire Protection Association.

National Fire Protection Association. (2015). NFPA 1003: Standard for Airport Fire Fighter Professional Qualifications. Quincy, MA: National Fire Protection Association.

Patton, M. Q. (2002). Qualitative research and evaluation methods $\left(3^{\text {rd }}\right.$ Ed.). Thousand Oaks, CA: Sage Publications, Inc.

Rauter, G., Sigrist, R., Koch, C., Crivelli, F., Raai, M. v., Riener, R., \& Wolf, P. (2013). Transfer of complex skill learning from virtual to real rowing. PLoS One, 8(12). doi: http://dx.doi.org/10.1371/journal.pone.0082145

Reweti, S. (2014). PC-Based Aviation Training Devices for Pilot Training in Visual Flight Rules Procedures; Development, Validation and Effectiveness (Doctoral dissertation, Massy University, Palmerston North, New Zealand). Retrieved from http://mro.massey.ac.nz/bitstream/handle/10179/5454/02_whole.pdf?sequ ence $=2$

Yin, Robert K. (2012). Case Study Research Design and Methods (5 ${ }^{\text {th }}$ Ed.). Thousand Oaks, California: Sage Publications, Inc. 
Appendix

Interview Protocol

\section{Introduction:}

Thank you for agreeing to participate in this study, we sincerely appreciate it. As you know, the results of this research will be published in a peer-reviewed professional journal, as well as the ARFF News so they can be shared with potentially interested members and other stakeholders interested in the topic.

Just to summarize where we are:

--you have signed and returned the Informed Consent letter;

--We have provided the Confidentiality Agreement to you. There will be no reasonable way your identity can be correlated with your interview transcript by anyone other than me. Your identity will a "number" coded to your name stored in a separate, password-protected electronic location. We will keep all study materials for a period of 5 years;

--you have the option to withdraw your participation at any time without adverse consequences to you;

--you have not received any inducements or payment for your participation in the study;

--and upon completion of the study, we will provide you with a summary of the research findings. It is our hope that the findings from this research can be used by the aircraft rescue and firefighting community. For the purposes of ensuring trustworthiness, this interview will be audio recorded. Do you have any questions up to this point?

\section{Interview}

This mixed methods study is intended to explore the experiences of users and instructors on the DFW FTRC interactive software intended to augment hands-on aircraft rescue and firefighting training. There are a series of 7 specific questions, but please feel free to share any and all information you think is important relative to the study. The questions, themselves, are not intended to constrain anything you wish to share in this interview related to the efficacy and use/potential use of the software.

First let's establish a context for you, as a key informant:

Name, length of service in work capacity, specific job duties/decisionmaking responsibilities related to the software/instruction. Do you feel you have any bias that should be noted in the study?

Now we will proceed to the interview questions:

1. What have you observed, in terms of the level of difficulty for students using the software? 
2. How well-integrated do you feel the software is within the curriculum? What changes would you recommend to better leverage the software's capabilities?

3. Have you observed that the software in the classroom augments the handson portion of the training? How?

4. Do you believe the software creates a better learning experience for students? Why or why not?

5. To follow up on the quantitative survey administered by the FTRC last year, we have a question to get the instructor perspective:

a) On the question "how would your department/agency use the software, $55 \%$ of respondents indicated strategies and tactics, $37 \%$ aircraft familiarization, and $8 \%$ airport familiarization; from what you have seen and heard from students, how might you explain this?

6. What recommendations for improvements to the software itself, integration into the classroom, and/or the delivery method would you suggest moving forward?

7. Do you think this type of software should be part of ARFF training no matter where the students receive their training?

At this time, I would like to ask you if there is anything you think is important to understanding your experience with the phenomenon that we have not yet discussed?

\section{Conclusion:}

We very much appreciate your taking the time to meet with us today to discuss your particular experience with the software. Your unique perspective will help the ARFF community and fire service professionals, like yourself.

As we mentioned previously, your interview responses will be kept completely confidential and you may withdraw from the study at any time. We will be sending you a summary of the key themes which emerged from this interview so that you can clarify your responses if we have misinterpreted them. We also appreciate your willingness to answer any follow-up questions which may arise as we begin data analysis. Upon completion of the research, we will send you a summary of the findings. Thank you very much for your participation. 